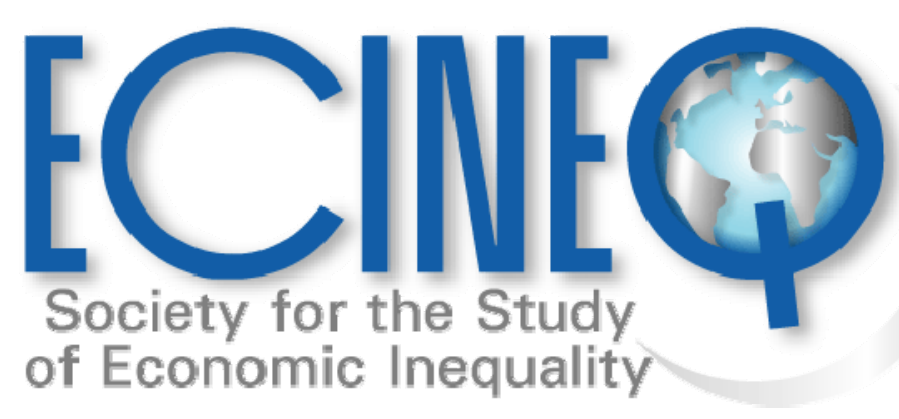

Working Paper Series

The Measurement of Rank Mobility

Marcello D'Agostino

Valentino Dardanoni 


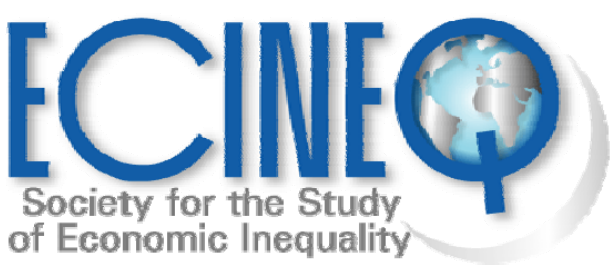

ECINEC 2007-80

November 2007

www.ecineq.org

\title{
The Measurement of Rank Mobility
}

\author{
Marcello D'Agostino \\ Università di Ferrara \\ Valentino Dardanoni* \\ Università di Palermo
}

\begin{abstract}
In this paper we investigate the problem of measuring social mobility when the social status of individuals is given by their rank. In order to sensibly represent the rank mobility of subgroups within a given society, we address the problem in terms of partial permutation matrices which include standard ("global") matrices as a special case. We first provide a characterization of a partial ordering on partial matrices which, in the standard case of global matrices, coincides with the well-known "concordance" ordering. We then provide a characterization of an index of rank mobility based on partial matrices and show that, in the standard case of comparing two global matrices, it is equivalent to Spearman's index.
\end{abstract}

JEL Classification: D31, D63.

Keywords: Mobility measurement, Concordance, Partial matrices, Sperman's Index. 


\title{
THE MEASUREMENT OF RANK MOBILITY
}

\author{
MARCELLO D'AGOSTINO AND VALENTINO DARDANONI
}

\begin{abstract}
In this paper we investigate the problem of measuring social mobility when the social status of individuals is given by their rank. In order to sensibly represent the rank mobility of subgroups within a given society, we address the problem in terms of partial permutation matrices which include standard ("global") matrices as a special case. We first provide a characterization of a partial ordering on partial matrices which, in the standard case of global matrices, coincides with the well-known "concordance" ordering. We then provide a characterization of an index of rank mobility based on partial matrices and show that, in the standard case of comparing two global matrices, it is equivalent to Spearman's $\rho$ index.
\end{abstract}

KEYWORDS: Mobility measurement, Concordance, Partial matrices, Sperman's index.

JEL numbers: D31, D63.

\section{INTRODUCTION}

When discussing social mobility issues, a basic distinction is usually made between intergenerational mobility (how the distribution of some relevant measure of individual status changes between different generations in a given society) and intragenerational mobility (how the distribution of individual status changes among a group of individuals over a given period of their lifetime). As a vehicle of discussion, we shall concentrate on intergenerational mobility, but all our considerations and results could be easily transposed to the intragenerational case. All the information about a social mobility context is then contained in a bivariate cumulative distribution function, which describes the distribution of two random variables capturing fathers' and sons' socio-economic status. It is widely believed that socioeconomic mobility is somewhat an elusive concept, difficult to define, let alone to measure: as remarked by Fields and Ok [FO99a] in a recent survey "... the mobility literature does not provide a unified discourse of analysis. ... a considerable rate of confusion confronts a newcomer in the field." ${ }^{1}$ This may be contrasted with the literature on income inequality, where a consensus has emerged on what concepts of inequality mean, on the correct theoretical procedures to measure it, and on how to go from theory to empirical applications.

Date: 8 September 2007.

${ }^{1}$ See also Maasoumi [Maa98] for a survey on mobility measurement. 
One of the main challenges in mobility measurement is the precise definition of individual socio-economic status, and its practical implementation using available data. Typically, mobility studies use data such as current or permanent income, consumption, occupational prestige, education etc. For ease of presentation, assume that income data is used for this purpose. In this paper we explore the possibility that the socioeconomic status of each individual is given by his rank, i.e. by its relative position in the generation to which he belongs. This way of defining individual status seems quite natural and intuitively satisfying. Indeed, the Encyclopedia Britannica defines social status as "the relative rank that an individual holds" and some analysts follow this common-sense interpretation by equating the concept of social status with that of rank. However, although being an interesting and much used notion, to the best of our knowledge rank mobility has not been thoroughly investigated so far, and this paper attempts to fill this gap.

To be precise, suppose $\left(x_{1}, y_{1}\right), \ldots,\left(x_{n}, y_{n}\right)$ describe the population distribution of fathers' and sons' incomes in a society of $n$ families. We make the simplifying assumption that there are no ties in the marginal distributions. ${ }^{2}$ Then, for our purposes, the socio-economic status of the $i$ th father is given by the number of fathers with income less than or equal to $y_{i}$, and the the socio-economic status of the $j$ th son is given by the number of sons with income less than or equal to $x_{j}$. Hence, rank mobility comparisons use only the information on the order of the $x$ 's and $y$ 's. Mobility comparisons which use cardinal information on fathers' and sons' income are axiomatized, among others, by [CBW85, Cow85, FO96, FO99b, GS02, Kin83, MZ86, MO98, RC04] and capture different aspects of social mobility than the present paper. These contributions may be considered more complementary than alternative to our approach. Fields ([Fie02], chapter 6) compares some theoretical properties of various indices of income mobility including some indices of rank mobility, and Buchinsky et. al. [BFFK05] compare their empirical properties in an application to French income mobility.

Given our assumption of no ties in the marginal distributions, all the information concerning the rank mobility of a society is contained in a permutation matrix $P$, with typical element $P(i, j)$ equal to 1 if there is a family in this society whose father has rank $i$ and son has rank $j$, and 0 otherwise. The problem is how to turn this information into a quantitative measure. ${ }^{3}$ In order to achieve a faithful and consistent representation of the rank mobility of subgroups within a given society, we address the problem in terms of partial permutation matrices (defined in Section 2) which include standard ("global") matrices as a special case, and argue that a representation of the rank mobility of a given subgroup of the population in terms of global matrices would be

\footnotetext{
${ }^{2}$ This case typically arises when $\left(x_{1}, y_{1}\right), \ldots,\left(x_{n}, y_{n}\right)$ is actually a random sample generated from a continuous distribution (so that ties occur with probability zero).

${ }^{3}$ Note that if $\left(x_{1}, y_{1}\right), \ldots,\left(x_{n}, y_{n}\right)$ is a random sample and we multiply by $\frac{1}{n}$ the permutation matrix $P$ we obtain the so called empirical joint rank distribution function (see Block et al. [BCFS90]).
} 
paradoxical. After observing that a standard decomposability property — which is key in the characterization of additively separable indices - cannot be sensibly assumed in the context of measuring rank mobility, we take advantage of our representation in terms of partial matrices to define a weaker form of decomposability which can be safely assumed. As an intermediate step, in section 3 we provide (Theorem 1) a characterization of a partial ordering on partial matrices which in the case of global matrices coincides with the well-known "concordance" ordering. We then provide (in Theorems 2 and 3) a rather natural and simple characterization (up to a monotonic transformation) of an index of rank mobility based on partial matrices and show that, in the standard case of comparing the mobility of two populations, this is equivalent to Spearman's $\rho$ index. Our characterization seems to provide reasonable grounds for adopting this kind of index (rather than other alternative ones, such as Kendall's $\tau$ or Spearman's footrule) in the measurement of rank mobility.

\section{Subgroup mobility and PARTial Permutation matrices}

Let $F$ denote the set of all families who live in a given society and consider a subset $A \subseteq F$; examples of interesting subsets are the families which live in a given geographical location, or which belong to a given race, or whose fathers have a given education level etc. Sometimes we may be interested in exploring how the status of individuals change from one generation to the next for members of this particular subset. We could call this kind of information the rank mobility of $A$ with respect to $F$. Observe that this is not the same as considering the rank mobility of $A$ w.r.t. $A$, because individuals' rank is calculated with respect to the whole of $F$. A simple example may help to clarify. Consider a society $F$ consisting of six families in which the distributions of fathers' and sons' incomes is summarized in Table 1 below:

\begin{tabular}{l|cccccc} 
& 1 & 2 & 3 & 4 & 5 & 6 \\
\hline Fathers & 100 & 150 & 200 & 250 & 300 & 350 \\
Sons & 150 & 200 & 100 & 250 & 350 & 300
\end{tabular}

TABLE 1. Incomes of fathers and sons in $F$.

Now, consider the subset $A$ of $F$ consisting only of the third, fourth and fifth families. If we consider only $A$ and calculate the rank of individuals with respect to this specific subset, then there is no rank mobility from one generation to the next. Viceversa, there is clearly a sense in which the families in $A$ exhibit some status mobility, which is made apparent when the status is calculated with respect to the whole of $F$ : the son of the third family has lost two positions with respect to his generation, while the son of the fifth family has gained one position.

This kind of "partial" mobility information, i.e. restricted to a subset of a whole set $F$ of families, will then be described by an $n \times n$ matrix which differs from a 
permutation matrix because it can have rows and columns with zeros only. Such matrices are called partial permutation matrices. ${ }^{4}$ When necessary for clarity, we shall call ordinary permutation matrices global. More formally, the set $\mathcal{P}_{n}$ of $n \times n$ partial permutation matrices is defined as follows: a matrix $P$ belongs to $\mathcal{P}_{n}$ if and only if, for all $i=1, \ldots, n$ and $j=1, \ldots, n$, we have: (i) $P(i, j) \in\{0,1\}$; (ii) $\sum_{i} P(i, j) \leq 1$; (iii) $\sum_{j} P(i, j) \leq 1$. Notice that, under this definition, global matrices are nothing but a special case of partial matrices.

Now, suppose $A$ and $B$ are disjoint subsets of a set $F$ of $n$ families. Clearly the partial permutation matrices that describe the rank mobility of $A$ and $B$ with respect to $F$, call them $P$ and $Q$, will belong to $\mathcal{P}_{n}$ and will be disjoint in a related sense that is expressed by the following definition:

Definition 1. $P, Q \in \mathcal{P}_{n}$ are disjoint if

$$
P(i, j)=1 \text { and } Q(m, k)=1 \Rightarrow i \neq m \text { and } j \neq k \text {. }
$$

Corollary 1. $P, Q \in \mathcal{P}_{n}$ are disjoint if and only if $P+Q \in \mathcal{P}_{n}$, where + is the usual sum of matrices.

Therefore, the rank mobility with respect to $F$ of disjoint subsets of $F$ is represented by disjoint partial matrices. Notice that if we partition $F$ into $m$ mutually exclusive and exhaustive subsets $A_{1}, \ldots, A_{m}$, the rank mobility of these subsets with respect to $F$ will be described by mutually disjoint partial matrices $P_{1}, \ldots, P_{m}$ such that $P=$ $P_{1}+\cdots+P_{m}$. Let us say that a partial matrix $P$ is atomic if there exists exactly one $i$ and one $j$ such that $P(i, j)=1$, that is, if we are considering a subset containing exactly one family. We shall use the lower case letter $p$ (possibly with subscripts) to denote atomic matrices. Clearly, any partial permutation matrix in $\mathcal{P}_{n}$ will be equal to a sum $p_{1}+\cdots+p_{k}$ of $k \leq n$ atomic matrices, where $k=n$ only for global matrices.

Observe that any $n \times n$ partial matrix $P$ can be regarded as representing the rank mobility of some set of families $A$ with respect to some "society" $F$ of size $n$ that includes it. Indeed, it is always possible to find an $F$ such that the rank mobility determined by the marginal distributions of fathers' and sons' income is represented by a global matrix that includes $P$. So, the above corollary implies that the sum of two disjoint partial matrices can always be regarded as representing the rank mobility of some suitable subgroup $A$ of a possible "society" $F$. Therefore, in this abstract setting, we can forget about "real" families, groups and societies and concentrate only on the partial matrices that represent their rank mobility. However, to avoid longwinded sentences, we shall often abuse of the more concrete terminology and speak, for instance, of "a society (group) $P$ " to mean "a society (group) whose rank mobility is represented by the (partial) matrix $P$ ", or of "the family $(i, j)$ (in a matrix $P$ )" to

\footnotetext{
${ }^{4}$ See e.g. Horn and Johnson [HJ91] for definitions and some properties of partial permutation matrices.
} 
mean "the family in which the father's rank is $i$ and the son's rank is $j$ (in a society whose rank mobility is represented by $P$ )".

We are interested in axiomatizing the properties of some suitable ordering $\preceq_{M}$ over $\mathcal{P}_{n}$ such that $P \preceq_{M} Q$ can be taken as meaning that the matrix $Q$ exhibits at least the same degree of social mobility as the matrix $P$.

\section{Axiomatizing RANK MOBILITY ORDERINGS}

In this section we shall start investigating the ordering relation $\preceq_{M}$. Given two matrices $P$ and $Q$ in $\mathcal{P}_{n}$, when can we say that $Q$ displays at least the same rank mobility as $P$ ? We introduce and discuss some plausible axioms to impose on $\preceq_{M}$ and then derive characterization theorems following an incremental approach. As a first step, in Section 3.1, we shall only assume that $\preceq_{M}$ is a quasi-ordering, that is a reflexive and transitive binary relation on $\mathcal{P}_{n}$, and shall not assume that the ordering is complete. Then, we derive, in Theorem 1, a characterization of what we propose as the basic rank mobility ordering from two basic axioms. Next, in Section 3.2, we investigate the possible completions of this basic quasi-ordering, and add further axioms which allow us to obtain sharper characterizations in Theorems 2 and 3.

3.1. The concordance ordering. While it is intuitively clear that it is meaningful to compare two standard (i.e. global) permutation matrices representing the rank mobility of two societies $F$ and $F^{\prime}$, it is not quite as clear whether it is equally meaningful to compare partial matrices - representing, say, the rank mobility of some subset $A \in F$ w.r.t. $F$ and the rank mobility of some subset $B \in F^{\prime}$ w.r.t. $F^{\prime}$ - when they have different marginal distributions. ${ }^{5}$ We shall therefore start by restricting the comparison to a clear-cut case.

\section{Definition 2.}

(1) Two matrices $P, Q \in \mathcal{P}_{n}$ are similar if

$$
\begin{aligned}
& \{i \mid P(i, j)=1 \text { for some } j\}=\{i \mid Q(i, j)=1 \text { for some } j\} \text { and } \\
& \{j \mid P(i, j)=1 \text { for some } i\}=\{j \mid Q(i, j)=1 \text { for some } i\} .
\end{aligned}
$$

(2) A matrix $P \in \mathcal{P}_{n}$ is monotone if, for all $i, j, m, k$ such that $P(i, j)=1$ and $P(m, k)=1$, we have $(i-m)(j-k)>0$.

So, two matrices are similar when they have equal marginal distributions. Note that the definition of similarity induces an equivalence relation on $\mathcal{P}_{n}$. Moreover, observe that within each similarity set there is a unique monotone matrix which can be considered as displaying the least amount of mobility:

\footnotetext{
${ }^{5}$ Loosely speaking, by "marginal distributions" of a partial matrix $P$ we mean the following: the marginal distribution of the fathers is the set of all $i$ such that $P(i, j)=1$ for some $j$, and the marginal distribution of the sons is the set of all $j$ such that $P(i, j)=1$ for some $i$.
} 
Axiom 1 (Monotonicity). For any distinct $P, Q \in \mathcal{P}_{n}$ such that $P$ is monotone and similar to $Q$ we have $P \prec_{M} Q$.

Notice also that if a matrix is monotone, then, and only then, there is a strictly increasing function from fathers' rank to sons' rank. ${ }^{6}$

The second axiom requires that the sum of disjoint partial matrices is a monotonic operation:

Axiom 2 (Subgroup Consistency). For every $P_{1}, P_{2}, P_{3}, P_{4} \in \mathcal{P}_{n}$ such that $P_{1}$ is disjoint with $P_{2}$ and $P_{3}$ is disjoint with $P_{4}$

$$
P_{1} \preceq_{M} P_{3} \text { and } P_{2} \preceq_{M} P_{4} \Rightarrow P_{1}+P_{2} \preceq_{M} P_{3}+P_{4} .
$$

Similar axioms are commonly used in the literature on income inequality [Sho88], poverty [FS91] and mobility measurement [FO99b], ${ }^{7}$ where they usually imply a fundamental, and practically useful, decomposability property: given an arbitrary partition of a population into $k$ subgroups, the problem of measuring a certain feature in the overall population can be reduced to the $k$ separate problems of measuring that feature in each of the $k$ subgroups. ${ }^{8}$ It must be stressed that the above axiom cannot be interpreted as asserting a similar decomposability of the rank mobility of a society $F$ into the rank mobility of their subgroups. In the terminology used in the introduction, this would amount to asserting that, given a partition of $F$ into $A_{1}, \ldots, A_{k}$, the rank mobility of $F$ w.r.t. $F$, can be decomposed into the rank mobility of $A_{1}$ w.r.t. $A_{1}, A_{2}$ w.r.t. $A_{2}$, etc., where the rank of each individual is evaluated with reference to the subgroup to which it belongs. However, this would clearly be paradoxical in the context of measuring rank mobility.

To see why, recall the simple example given in Section 2 (see Table 1), considering a society made of six families. Let $A_{1}$ be the subgroup consisting of the third, fourth and fifth families, and $A_{2}$ the subgroup consisting of the first, second and sixth. Now, it is clear that the rank mobility of the whole $F$ w.r.t. $F$ is greater than zero, while the rank mobility of $A_{1}$ w.r.t. $A_{1}$ and the rank mobility of $A_{2}$ w.r.t. $A_{2}$ are both, intuitively, equal to zero. Hence, we cannot hope that rank mobility enjoys such a strong decomposability property. ${ }^{9}$ However, our axiom states a weaker decomposability

\footnotetext{
${ }^{6}$ The reader may find it helpful to compare our concept of similar matrices with the well-known Frechet class of distributions with fixed marginals, and our monotonicity axiom with the lower bound in the Frechet class (see e.g. Nelsen [Nel99]).

${ }^{7}$ Though such axioms are widely accepted in these contexts, for a critical discussion see Foster and Sen [FS97].

${ }^{8}$ For this interpretation of the decomposability property in the context of social mobility see, for instance, Fields and Ok [FO96]. The term decomposability has different interpretations in other theoretical and applied contexts.

${ }^{9}$ Tha a mechanical application of standard decomposability properties is clearly nonsensical in this context is remarked, for example, by Cowell [Cow85] page 144, who explicitly states that distance measures based on ranks are not decomposable.
} 
property: given a partition of $F$ into $A_{1}, \ldots, A_{n}$, the rank mobility of $F$ w.r.t. $F$, can be decomposed into the rank mobility of $A_{1}$ w.r.t. $F, A_{2}$ w.r.t. $F$, etc., where the rank of each individual is evaluated with reference to the whole society $F$. Accordingly, the axiom is expressed in terms of partial permutation matrices for a society $F$, which are obtained from the global permutation matrix for $F$ by omitting information concerning some of the families, and are intended to represent the rank mobility of given subgroups with respect to the whole of $F$. Our subgroup consistency axiom, therefore, cannot be interpreted as allowing us to measure the rank mobility of a population in terms of independent measurements of the rank mobility of its subgroups. Indeed, our axiom is better understood as a monotonicity requirement on the sum of disjoint partial permutation matrices, and, from this point of view, it expresses a basic logical property that seems quite uncontroversial.

Suppose, now, that we have a matrix $P$ such that, for the indices $k<m$ and $l<n$ we have $P(k, l)=P(m, n)=1$, and consider another matrix $Q$ such that $P(i, j)=P(i, j)$ for all $i \neq k, m$ and $j \neq l, n$, and $Q(k, n)=Q(m, l)=1$. In words, $Q$ differs from $P$ because there has been an inversion of social status between two families, such that before the inversion the higher status father had the higher status son, while after the inversion the lower status father has the higher status son. Intuitively, such an inversion should be mobility-increasing. ${ }^{10}$ Under these circumstances we say that $Q$ has been obtained from $P$ by inverting $(k, l)$ and $(m, n)$. We write $P \prec Q$ whenever $Q$ can be obtained from $P$ by means of such an inversion.

Suppose, a given matrix $Q$ can be obtained from $P$ by a sequence of inversions. We can intuitively conclude that $Q$ displays more social mobility than $P$, and write $P \preceq_{C} Q$. Formally:

Definition 3. $P \preceq_{C} Q$ if and only if there is a finite sequence of matrices $P_{0}, \ldots, P_{k}$, with $k \geq 0$, such that (i) $P_{0}=P$, (ii) $P_{k}=Q$ and (iii) if $k>0, P_{i-1} \prec P_{i}$ for all $i=1, \ldots, k$.

It can be easily checked that $\preceq_{C}$ is a partial order ${ }^{11}$ defined on each set of similar matrices. The reason for the choice of the subscript " $C$ " is that, when the similarity class consists of the global matrices in $\mathcal{P}_{n}, \preceq_{C}$ is called the concordance ordering in the mathematical statistics literature, see e.g. Tchen [Tch80] and Kimeldorf and Sampson [KS87].

Theorem 1. Within each set of similar matrices, $\preceq_{C}$ is the smallest ${ }^{12}$ quasi-ordering which satisfies Axiom 1 and Axiom 2.

\footnotetext{
${ }^{10}$ Such swaps are well-known in the mathematical statistics [Tch80] and economics [ET80, Atk83, Dar93] literature and it is often assumed that they are always mobility-increasing.

${ }^{11}$ That is, besides being reflexive and transitive, it is also antysimmetric, namely: $P \preceq_{C} Q$ and $Q \preceq_{C} P$ imply that $P=Q$.

${ }^{12}$ In terms of set-inclusion.
} 
A proof of this theorem is given in Appendix A. The concordance ordering $\preceq_{C}$ is a very well established and much studied ordering of bivariate distributions. Atkinson [Atk83] first applies the concordance ordering to mobility measurement; Dardanoni [Dar93] applies it to a Markov chain model of social mobility, and shows the equivalence of a version of this ordering to some very intuitive concepts of greater social mobility. It is a partial ordering which, in the space of global permutation matrices, is a subrelation of many important complete orders, for example, those induced by the popular nonparametric indices of concordance such as Kendall's $\tau$ and Spearman's $\rho$, see e.g. Schweizer and Wolff [SW81]. The theorem then says that all reflexive and transitive relations $\preceq_{M}$ which satisfy Axioms 1 and 2 must have a common area of agreement equal to $\preceq_{C}$.

On the other hand, $\preceq_{C}$ allows for comparisons between similar matrices only and, while this restriction is immaterial when comparing global matrices, it makes the comparison of partial matrices impossible except for the artificial special case in which the matrices have exactly the same marginal distributions. Moreover, being a partial ordering, $\preceq_{C}$ does not even allow for comparisons of all matrices in a given similarity set. Thus, in order to be able to compare all mobility contexts in $\mathcal{P}_{n}$, we must focus on weak orderings, ${ }^{13}$ rather than quasi-orderings. Clearly, even assuming that $\preceq_{M}$ is a weak ordering, Axioms 1 and 2 are not sufficient to uniquely characterize it (since there are several weak orderings, e.g. the above mentioned $\rho$ and $\tau$, that satisfy them.) From this point of view, Theorem 1 only implies that every weak ordering $\preceq_{M}$ that satisfies the axioms must include $\preceq_{C}$, and so the properties expressed by the axioms can be considered as minimal requirements on any suitable mobility ordering. So, in the sequel, we shall take our mobility ordering $\preceq_{M}$ to be a weak ordering and seek for extra axiomatic properties that allow us to uniquely characterize it.

3.2. Completing the concordance ordering. In this section we investigate the possible completions of the basic concordance ordering characterized in Theorem 1. We shall therefore assume that our mobility ordering $\preceq_{M}$ is a weak (i.e. transitive and complete) ordering and consider the class of weak orderings $\preceq_{M}$ satisfying Axioms 1 and 2. (As implied by Theorem 1, they must all include the concordance ordering.) Our aim, now, is to investigate how these axioms can be expanded to single out a suitable mobility ordering from this class.

There are two distinct intuitive aspects of the notion of "greater mobility" which emerge from its conceptual analysis. One aspect, which is apparent in the standard definitions of some well-known orderings - such as the concordance ordering and the weak ordering based on Kendall's function $\tau$ - stems from the idea that there is an increase in mobility when two families interchange their relative position. On the other hand, from a different angle, mobility is related to the distance between father's and

\footnotetext{
${ }^{13}$ Recall that a weak ordering is a quasi-ordering which is also complete, namely such that, for all $P, Q \in \mathcal{P}_{n}$, either $P \preceq_{M} Q$ or $Q \preceq_{M} P$.
} 
sons's status within each family, and overall mobility of a group of families may be construed as the aggregation of the degrees of mobility exhibited by all the families in that group. ${ }^{14}$

Now, for a single family in a society $F$, such that father's rank is $i$ and son's rank is $j$, we can take $|i-j|$ as measuring the social distance between father's and son's social status. This basic intuition is captured by the following:

Axiom 3 (Atomic Monotonicity). For any two atomic matrices $p, q \in \mathcal{P}_{n}$ such that $p(i, j)=q\left(i^{\prime}, j^{\prime}\right)=1$,

$$
p \preceq_{M} q \Longleftrightarrow|i-j| \leq\left|i^{\prime}-j^{\prime}\right| .
$$

Notice that, although this axiom forces a unique weak ordering of atomic matrices, it is not sufficient to uniquely characterize $\preceq_{M}$ in the whole of its domain.

Let's now introduce some notation which will simplify considerably the following discussion. If " $P$ " denotes a matrix in a given space $\mathcal{P}_{n}$, then " $P$ " ", with $m \geq n$, will denote the matrix in $\mathcal{P}_{m}$ which coincides with $P$ wherever $P$ is defined and contains only 0's everywhere else, i.e. the matrix defined as follows:

$$
\begin{aligned}
& P^{m}(i, j)=P(i, j) \text { for all } i, j \leq n \text { and } \\
& P^{m}(i, j)=0 \text { for any } i, j \text { such that } i>n \text { or } j>n .
\end{aligned}
$$

On the other hand, if " $P$ " denotes a matrix in $\mathcal{P}_{m}$, we shall attach no meaning to the notation " $P$ " " with $k<m$. Observe that, by definition,

(1) $\left(P^{k}\right)^{m}=P^{m}$ for every $m \geq k$

(2) $(P+Q)^{m}=P^{m}+Q^{m}$ for every disjoint $P, Q \in \mathcal{P}_{k}$ with $k \leq m$.

Let us say that a matrix $P$ is null if $i=j$ for all $(i, j)$ such that $P(i, j)=1$. Intuitively, a null-matrix says that the subgroup for which it is defined displays no mobility at all. The following axiom is an adaptation of the well-known Archimedean Property to our setting:

Axiom 4 (Archimedean Property). For every $m$ and all $P, Q \in \mathcal{P}_{m}$, the strict inequality $P \prec_{M} Q$ holds if and only if there is an $n \geq m$ and a non-null $R \in \mathcal{P}_{n}$, disjoint with $P^{n}$, such that

$$
P^{n}+R \sim_{M} Q^{n}
$$

Observe that, within a given space $\mathcal{P}_{n}$, a partial permutation matrix $P$ is uniquely determined by the set $S(P)=\{(i, j) \mid P(i, j)=1\}$. We call $S(P)$ the characteristic set of $P$.

Now, we can prove the following:

\footnotetext{
${ }^{14}$ Clearly these two concepts of mobility (one which considers the interplay of families and the other which considers families in isolation) are interrelated, since single families cannot change relative positions without affecting other families.
} 
Theorem 2. $\preceq_{M}$ is a weak ordering satisfying Axioms 1-4 if and only if there is a strictly increasing and strictly convex function $f: \mathbb{N} \rightarrow \mathbb{N}$ such that, for all $n$ and for all $P, Q \in \mathcal{P}_{n}$,

$$
P \preceq_{M} Q \Longleftrightarrow \sum_{(i, j) \in S(P)} f(|i-j|) \leq \sum_{(i, j) \in S(Q)} f(|i-j|) .
$$

A proof of this theorem is given in Appendix B. Theorem 2 shows that Axioms 14 characterize (up to a monotonic transformation) a class of additive mobility indices which depends on the choice of an appropriate weighting function $f$. It is interesting to notice that, within the space of global matrices, two important indices of ordinal association which would seem appropriate to (im)mobility measurement, namely Kendall's $\tau$ and Spearman's footrule (see e.g. Kendall and Gibbons [KG90] for definitions and a discussion of their properties) do not belong to the class defined in Theorem 2 .

Consider for example the global permutation matrices $P, P^{\prime}$ and $P^{\prime \prime}$ in $\mathcal{P}_{4}$ with the following characteristic sets:

$$
\begin{aligned}
S(P) & =\{(1,1),(2,4),(3,3),(4,2)\} \\
S\left(P^{\prime}\right) & =\{(1,3),(2,1),(3,4),(4,2)\} \\
S\left(P^{\prime \prime}\right) & =\{(1,1),(2,3),(3,4),(4,2)\} .
\end{aligned}
$$

Using any of the mobility indices, say $M$, in the class characterized by Theorem 2 , the mobility of $P, P^{\prime}$ and $P^{\prime \prime}$ will be equal to

$$
\begin{aligned}
M(P) & =H(f(0)+f(2)+f(0)+f(2)), \\
M\left(P^{\prime}\right) & =H(f(2)+f(1)+f(1)+f(2)), \\
M\left(P^{\prime \prime}\right) & =H(f(0)+f(1)+f(1)+f(2))
\end{aligned}
$$

for some strictly increasing and strictly convex $f$ and strictly increasing $H$. Now, if we adopted Spearman's footrule as a mobility measure, which corresponds to letting $f$ be the identity function, $P$ and $P^{\prime \prime}$ would display the same amount of mobility, since $0+2+0+2=0+1+1+2$. However, $P$ can be derived from $P^{\prime \prime}$ by an inversion of the families $(2,3)$ and $(3,4)$. Thus $S^{\prime \prime} \prec_{C} S$, and so Spearman's footrule is inconsistent with $\preceq_{C}$. This failure of Spearman's footrule to satisfy the basic ordering $\preceq_{C}$ makes it unsuitable for measuring rank mobility.

On the other hand, it is easy to show that, in the class of global matrices, Kendall's $\tau$ does indeed agree with $\preceq_{C}$ (see e.g. Schweizer and Wolff [SW81]). Nevertheless, it cannot satisfy all our axioms, as can be seen by observing that $P$ and $P^{\prime}$ have the same value of Kendall's $\tau$, while any of the indices of Theorem 2 would deliver different values, since in $P^{\prime}$ there are two families with social distance equal to 2 (as in $P$ ), but, in addition, there are also two families with positive social distance (since $f$ is strictly increasing). 
Now, while Theorem 2 characterizes a class of mobility indices which is small enough to exclude some important indices, it clearly contains more than one possible mobility index, so that its practical application is still dependent on the choice of the function $f$. This choice can, however, be guided by the following argument: consider an inversion of two families $(i, j)$ and $(i+1, j+1)$, so that 1 is the distance between the fathers and 1 is the distance between the sons before the inversion. We shall write $P \triangleleft Q$ when $Q$ is obtained from $P$ by means of such an inversion.

It may be reasonable to assume that all the inversions of this type are minimal inversions and that they all generate an equivalent mobility increase. ${ }^{15}$ This assumption is expressed by the following axiom:

Axiom 5 (Minimal Inversion). For any matrices $P, Q, R, \in \mathcal{P}_{n}$,

$$
P \triangleleft Q \text { and } P \triangleleft R \Longrightarrow Q \sim_{M} R \text {. }
$$

Then we can prove the following:

Theorem 3. $\preceq_{M}$ is a weak ordering satisfying Axioms 1-5 if and only if for any $P, Q \in \mathcal{P}_{n}$,

$$
P \preceq_{M} Q \Longleftrightarrow \sum_{(i, j) \in S(P)}(i-j)^{2} \leq \sum_{\left(i^{\prime}, j^{\prime}\right) \in S(Q)}\left(i^{\prime}-j^{\prime}\right)^{2} .
$$

A proof is given in Appendix C. It can be easily verified that, within the set of global matrices, Theorem 3 characterizes (up to a monotonic transformation) the well-known Spearman index of ordinal association, since the latter (which is better described as an immobility index) can be written as

$$
\rho(P)=1-\frac{6 \sum_{(i, j) \in S(P)}(i-j)^{2}}{n^{3}-n}
$$

(see e.g. Kendall and Gibbons [KG90], page 8).

On the other hand, the ordering characterized in Theorem 3 is not restricted to populations' comparisons. For partial permutation matrices, the theorem provides a means for comparing the status mobility of different subgroups when the concept of social status we are interested in refers to the rank of individuals in the whole society. As an example, recall again the society $F$ considered in in Section 2 (see Table 1), and assume that the third, fourth and fifth family belong to a first group, while the first, second and sixth belong to a second group. It is then easily calculated that families in the first group exhibit a greater level of rank mobility than those in the second, since applying Theorem 3 we have $4+0+1>1+1+1$.

\footnotetext{
${ }^{15}$ By contrast, we might assume that the increase of mobility generated by an inversion should depend, somehow, also on the values of the father's rank $i$ and the son's rank $j$ in the inverted families. In this case, of course, not all minimal inversions would be equivalent.
} 


\section{APPENDICES}

\section{Appendix A. Proof of Theorem 1}

Proof. We first show that the $\preceq_{C}$ ordering (which, we recall, is defined on each set of similar partial matrices) satisfies the axioms. It is obvious that it satisfies Axiom 1. As for Axiom 2, suppose $P_{1}, P_{2}$ and $P_{3}, P_{4}$ are mutually disjoint, and $P_{1} \preceq_{C} P_{3}$ and $P_{2} \preceq_{C} P_{4}$. Then there exists a sequence $Q_{0}, \ldots, Q_{k}$ of partial matrices in $\mathcal{P}_{n}$ such that (i) $Q_{0}=P_{1}$, (ii) $Q_{k}=P_{3}$ and (iii) $Q_{i} \prec Q_{i+1}$ for $i=0, \ldots, k-1$. Similarly, there exists a sequence $R_{0}, \ldots, R_{k^{\prime}}$ of partial matrices in $\mathcal{P}_{n}$ such that (i) $R_{0}=P_{2}$, (ii) $R_{k^{\prime}}=P_{4}$ and (iii) $R_{i} \prec R_{i+1}$ for $i=0, \ldots, k^{\prime}-1$. Suppose $k^{\prime}>k$. Then, it is easy to see that, since $P_{1}, P_{2}$, and $P_{3}, P_{4}$ are mutually disjoint, the sequence

$$
Q_{0}+R_{0}, \ldots, Q_{k}+R_{k}, Q_{k}+R_{k+1}, \ldots, Q_{k}+R_{k^{\prime}}
$$

is such that (i) $Q_{0}+R_{0}=P_{1}+P_{2}$, (ii) $Q_{k}+R_{k^{\prime}}=P_{3}+P_{4}$, (iii) $Q_{i}+R_{i} \prec Q_{i+1}+R_{i+1}$ for $i=0, \ldots, k-1$, and (iv) $Q_{k}+R_{j} \prec Q_{k}+R_{j+1}$ for $j=k, \ldots, k^{\prime}-1$. Hence, by definition of $\preceq_{C}$, we have that $P_{1}+P_{3} \preceq_{C} P_{2}+P_{4}$. The argument is similar when $k>k^{\prime}$.

Next, we show that if a quasi-ordering $\preceq_{M}$ satisfies the axioms, then it must include the concordance ordering. This is sufficient to conclude that $\preceq_{C}$ is the smallest quasiordering satisfying the axioms.

Suppose $\preceq_{M}$ satisfies the axioms. Let $P$ and $Q$ be two matrices such that $P \preceq_{C} Q$. By definition, this means that $P$ and $Q$ are similar and there is a sequence of matrices $P_{0}, \ldots, P_{k}$, with $k \geq 0$, such that $P_{0}=P, P_{k}=Q$ and, if $k>0, P_{i} \prec P_{i+1}$ for all $i=$ $1, \ldots, k-1$. Now consider the $i$-th inversion step, and suppose it is such that, for some $j, m, l, n$ with $j<m$ and $l<n, P_{i-1}(j, l)=P_{i-1}(m, n)=1$ and $P_{i}(j, n)=P_{i}(m, l)=1$. Consider the matrix $P_{i-1}^{*}$ such that only $P_{i-1}^{*}(j, l)=P_{i-1}^{*}(m, n)=1$, while all the other entries are 0 (that is, its characteristic set $S\left(P_{i-1}^{*}\right)$ is equal to $\left.\{(j, l),(m, n)\}\right)$. Let also $P_{i}^{*}$ be the similar matrix such that only $P_{i}^{*}(j, n)=P_{i}^{*}(m, l)=1$, while all the others are 0 (that is, its characteristic set $S\left(P_{i}^{*}\right)$ is equal to $\{(j, n),(m, l)\}$ ).

Clearly

$$
P_{i-1}=\left(P_{i-1}-P_{i-1}^{*}\right)+P_{i-1}^{*} \text { and } P_{i}=\left(P_{i}-P_{i}^{*}\right)+P_{i}^{*} .
$$

Moreover, $P_{i-1}-P_{i-1}^{*}=P_{i}-P_{i}^{*}$ and, since $j<m$ and $l<n$, the matrix $P_{i-1}^{*}$ is a monotone matrix, so that $P_{i-1}^{*} \preceq_{M} P_{i}^{*}$ (by Axiom 1). Therefore,

$$
P_{i-1}=\left(P_{i-1}-P_{i-1}^{*}\right)+P_{i-1}^{*} \preceq_{M}\left(P_{i}-P_{i}^{*}\right)+P_{i}^{*}=P_{i}
$$

by Axiom 2. Hence, $P_{i-1} \preceq_{M} P_{i}$. The same argument holds for all $i$, therefore $P \preceq_{M} Q$. This shows that $P \preceq_{C} Q$ implies $P \preceq_{M} Q$ for all $P$, $Q$, i.e. $\preceq_{C}$ is included in $\preceq_{M}$. Since $\preceq_{M}$ was an arbitrary quasi-ordering satisfying the axioms, $\preceq_{C}$ is included in all the quasi-orderings satisfying the axioms. 


\section{Appendix B. Proof of Theorem 2}

B.1. Preliminary lemmas. The following lemmas turn out to be useful to make the proof of Theorem 2 more readable.

Lemma 1. If $\preceq_{M}$ is a weak ordering satisfying Axiom 4, then

$$
P \preceq_{M} Q \text { if and only if } P^{m} \preceq_{M} Q^{m},
$$

for all $n$, all $P, Q \in \mathcal{P}_{n}$ and all $m \geq n$.

Proof. Suppose $P \preceq_{M} Q$ and $P^{m} \swarrow_{M} Q^{m}$, that is $P^{m} \succ_{M} Q^{m}$, for some $m \geq n$. Then, by Axiom 4, there is an $m^{\prime} \geq m$ and a non-null $R \in \mathcal{P}_{m^{\prime}}$ such that $R$ is disjoint with $Q^{m^{\prime}}$ and

$$
Q^{m^{\prime}}+R \sim_{M} P^{m^{\prime}}
$$

Since $m^{\prime} \geq n$, it follows again from Axiom 4, that $Q \prec_{M} P$, against the hypothesis. Hence:

$$
P \preceq_{M} Q \Longrightarrow P^{m} \preceq_{M} Q^{m} .
$$

For the converse, suppose that for some $m>n, P^{m} \preceq_{M} Q^{m}$ and $P \succ_{M} Q$. Then, by Axiom 4,

$$
P^{k} \sim_{M} Q^{k}+R
$$

for some $k \geq n$ and some non-null $R \in \mathcal{P}_{k}$. Now, if $k \geq m$, this implies, again by Axiom 4, that $Q^{m} \prec_{M} P^{m}$ against the hypothesis. If $k<m$, it follows from (2), by (1), that $P^{m} \sim_{M}\left(Q^{k}+R\right)^{m} \sim_{M} Q^{m}+R^{m}$. Since $R^{m}$ is non-null, it follows, again by Axiom 4, that $Q^{m} \prec_{M} P^{m}$, against the hypothesis.

Lemma 2. If $\preceq_{M}$ is a weak ordering satisfying Axiom 4, then

$$
P \preceq_{M} P+R,
$$

for all $n$, and all $P, R \in \mathcal{P}_{n}$ such that $P+R \in \mathcal{P}_{n}$.

Proof. Suppose $P \succ_{M} P+R$. Then, by Axiom 4,

$$
P^{m} \sim_{M}(P+R)^{m}+S \sim_{M} P^{m}+R^{m}+S
$$

for some $m \geq n$ and some non-null $S \in \mathcal{P}_{m}$. Since $R^{m}+S$ is non-null, this would imply, again by Axiom 4, that $P \succ_{M} P$, which is impossible.

Let us denote by $\perp_{n}$ the unique matrix $P \in \mathcal{P}_{n}$, that we call the empty matrix, such that $P(i, j)=0$ for all $i, j$, that is the $n \times n$ matrix which is totally undefined. By definition, (i) $\perp_{n}$ is a null matrix (ii) for every $P \in \mathcal{P}_{n}, \perp_{n}$ is disjoint with $P$, and (iii) $\perp_{n}+P=P$. 
Lemma 3. If $\preceq_{M}$ is a weak ordering satisfying Axioms 2, 3 and 4, then

$$
\perp_{n} \sim_{M} P \prec_{M} Q
$$

for every null matrix $P \in \mathcal{P}_{n}$ and every non-null matrix $Q$ in $\mathcal{P}_{n}$.

Proof. First, recall that (by Axiom 4) $\perp_{n} \prec_{M} Q$, for every $n$ and every non-null $Q \in \mathcal{P}_{n}$, since $\perp_{n}+Q=Q \sim_{M} Q$. Hence, we only have to show that $P \sim_{M} \perp_{n}$ for every null $P \in \mathcal{P}_{n}$. If $P=\perp_{n}$, then it is trivially true that $P \sim_{M} \perp_{n}$. Consider, then, the case that $P \neq \perp_{n}$. Let us first show that $p \sim_{M} \perp_{n}$ for every null atomic matrix $p$. Suppose, that $p \succ_{M} \perp_{n}$. Then, by Axiom 4, $p^{m} \sim_{M} \perp_{m}+R=R$ for some $m \geq n$ and some non-null $R \in \mathcal{P}_{m}$. Since $R$ is non-null, $R=r+T$ for some non-null atomic matrix $r$ and some, possibly null, matrix $T$ in $\mathcal{P}_{m}$. By Axiom 3, $p^{m} \prec_{M} r$ and, by Lemma $2, p^{m} \prec_{M} r+T=R$. This is a contradiction, since we had before concluded that $p^{m} \sim_{M} R$. Suppose, then, that $\perp_{n} \succ_{M} p$. By Axiom 4, $p^{m}+R \sim \perp_{m}$ for some $m \geq n$ and some non-null $R \in \mathcal{P}_{m}$. Now, since $\perp_{m} \prec_{M} Q$ for every non-null $Q \in \mathcal{P}_{m}$, it follows that $\perp_{M} \prec_{M} R$. Then, by Lemma 3, $\perp_{M} \prec_{M} p^{m}+R$ against the previous conclusion that $\perp_{M} \sim_{M} p^{m}+R$. Hence, since $\preceq_{M}$ is a weak ordering, $\perp_{M} \sim_{M} p$.

If $P$ is not an atomic matrix and $P \neq \perp_{n}$, then $P=p_{1}+\cdots+p_{k}$ for some $k$ such that $1<k \leq n$, with each $p_{i}(1 \leq i \leq k)$ being a null atomic matrix. As we have just established, $p_{i} \sim_{M} \perp_{n}$ for all $i=1, \ldots, k$. Hence, by Axiom 2 (and recalling that $\left.\perp_{n}+\perp_{n}=\perp_{n}\right), P \sim_{M} \perp_{n}$.

Say that two matrices $P$ and $Q$ are atomically equivalent if, for every $k \geq 0$, they contain the same number of non-zero entries $(i, j)$ with $|i-j|=k$. Clearly, if $P$ and $Q$ are atomically equivalent, there are atomic matrices $p_{1}, \ldots, p_{m}$ and $q_{1}, \ldots, q_{m}$, with $m \leq n$, such that $P=p_{1}+\cdots+p_{m}, Q=q_{1}+\cdots+q_{m}$ and, by Axiom $3, p_{i} \sim_{M} q_{i}$ for $i=1, \ldots, m$. Hence, by Axiom 2, if $P$ and $Q$ are atomically equivalent, then $P \sim_{M} Q$.

Remark 1. Given any two matrices $P, Q \in \mathcal{P}_{n}$, one can always find a sufficiently large $m$ and a matrix $R$ in $\mathcal{P}_{m}$ such that $R$ is atomically equivalent to $Q^{m}$ and disjoint with $P^{m}$. For this purpose, it is sufficient to take $m=2 n$ and $R$ equal to the matrix such that (i) $R(i, j)=0$ for all $i, j \leq n$ and (ii) $R(n+i, n+j)=1$ if and only if $Q(i, j)=1$. Using this method, if $P_{1}, \ldots, P_{k}$ are matrices in $\mathcal{P}_{n}$, one can always find suitable matrices $P_{1}^{\prime}, \ldots, P_{k}^{\prime} \in \mathcal{P}_{k n}$ such that (i) $P_{i}^{k n} \sim_{M} P_{i}^{\prime}$ for $i \leq k$ and (ii) all the $P_{k n}^{\prime}$ are mutually disjoint.

Lemma 4. If $\preceq_{M}$ is a weak ordering satisfying Axioms 2 and 4 , then for all $n$, and all $P, Q, R, S \in \mathcal{P}_{n}$ such that $P$ is disjoint with $R$ and $Q$ is disjoint with $S$,

$$
P \sim_{M} Q \text { and } P+R \sim_{M} Q+S \Longrightarrow R \sim_{M} S
$$

Proof. Let us assume that $P \sim_{M} Q$ and $P+R \sim_{M} Q+S$. Suppose, ex absurdo, that $R \chi_{M} S$. 
Case 1: $R \succ_{M} S$. Then, it follows from Axiom 4, that $R^{m} \sim_{M} S^{m}+T$ for some $m \geq n$ and some non-null $T \in \mathcal{P}_{m}$. By Remark 1, there are $m^{\prime} \geq m$ and $U \in \mathcal{P}_{m^{\prime}}$ such that $U \sim_{M} P^{m^{\prime}}$ and $U$ is disjoint with $S^{m}+T$. Hence, by Lemma 1 and Axiom 2,

$$
P^{m^{\prime}}+R^{m^{\prime}} \sim_{M} U+S^{m^{\prime}}+T \sim Q^{m^{\prime}}+S^{m^{\prime}} .
$$

So, by Axiom 4, $U+S^{m^{\prime}} \prec_{M} Q^{m^{\prime}}+S^{m^{\prime}}$ (since $T$ is non-null). However, by Axiom 2, $U+S^{m^{\prime}} \sim_{M} Q^{m^{\prime}}+S^{m^{\prime}}$ (since $U \sim_{M} P^{m^{\prime}} \sim_{M} Q^{m^{\prime}}$ by hypothesis and Lemma 1), which is a contradiction.

Case 2: $R \prec_{M} S$. This case is similar to Case 1 and is left to the reader.

Now, consider the set $\mathcal{P}=\bigcup_{i=1}^{\infty} \mathcal{P}_{i}$ of all partial matrices. We define the subset $\Delta_{k}$, $k \geq 0$, of $\mathcal{P}$ as the set of all $P \in \mathcal{P}$ such that for all $(i, j) \in S(P),|i-j| \leq k$. Notice that $\Delta_{k} \subseteq \Delta_{m}$ whenever $k \leq m$. The matrices in $\Delta_{0}$ are the null matrices. We shall also write $\Delta_{k}^{n}$ for $\Delta_{k} \cap \mathcal{P}_{n}$. Moreover, given two matrices $P, Q \in \mathcal{P}_{n}$, let us say that $Q$ is contained in $P$ if $P(i, j)=1$ for all $i, j \in\{1, \ldots, n\}$ such that $Q(i, j)=1$. Recall that every partial matrix can be uniquely expressed as a sum of atomic matrices.

Lemma 5. If $\preceq_{M}$ is a weak ordering satisfying Axioms 2, 3 and 4, then for all $n$ and all $P, Q \in \Delta_{1}^{n}, P \preceq_{M} Q$ if and only if the number of non-null atomic matrices contained in $P$ is less than or equal to the number of non-null atomic matrices contained in $Q$.

Proof. Suppose first that the number of non-null atomic matrices contained in $P$ is less than or equal to the number of non-null atomic matrices contained in $Q$. Let $p_{1}, \ldots, p_{j}$ be the non-null atomic matrices in $P$ and $q_{1}, \ldots, q_{k}$ the non-null atomic matrices in $Q$, with $j \leq k \leq n$. Then $P=p_{1}+\cdots+p_{j}+R$ for some null $R \in \mathcal{P}_{n}$ and $Q=q_{1}+\cdots+q_{j}+S$ for some possibly non-null $S \in \mathcal{P}_{n}$. By Axiom 3 all the non-null atomic matrices in $\Delta_{1}^{n}$ are equivalent to each other and therefore, by Axiom $2, p_{1}+\cdots+p_{j} \sim_{M} q_{1}+\cdots+q_{j}$. Moreover, by Lemma $3, R \preceq_{M} S$. Hence, again by Axiom $2, P \preceq_{M} Q$. Suppose now that the number of non-null atomic matrices in $P$ is strictly greater than the number of non-null atomic matrices in $Q$. Let $p_{1}, \ldots, p_{k}$ the non-null atomic matrices in $P$ and $q_{1}, \ldots, q_{j}$ the non-null atomic matrices in $Q$, with $j \leq k \leq n$. So, $P=p_{1}+\cdots+p_{j}+R$ for some non-null $R \in \mathcal{P}_{n}$ and $Q=q_{1}+\cdots+q_{j}+S$ for some null $S \in \mathcal{P}_{n}$. Moreover, by Lemma 3, $S \prec_{M} R$ and, as argued above, $p_{1}+\cdots+p_{j} \sim_{M} q_{1}+\cdots+q_{j}$. So, by Axiom $2, Q=q_{1}+\cdots+q_{j}+S \preceq_{M} p_{1}+\cdots+p_{j}+R=P$. By Lemma $4, Q \sim_{M} P$ would imply that $S \sim_{M} R$ which, given that $S$ is null and $R$ is non-null, is ruled out by Lemma 3. Therefore, we can conclude that $Q \prec_{M} P$.

Lemma 6. If $\preceq_{M}$ is a weak ordering satisfying Axioms 2-4, then for every $n$ and every atomic matrix $p \in \mathcal{P}_{n}$, there is an $m \geq n$ such that $p^{m} \sim_{M} Q$ for some $Q \in \Delta_{1}^{m}$.

Proof. Since every atomic matrix $p \in \mathcal{P}_{n}$ belongs to some $\Delta_{k}^{n}$, with $k \in \mathbb{N}$, we prove the lemma by induction on the index $k$ of the smallest class $\Delta_{k}^{n}$ to which $p$ belongs. In the course of the proof, and for the sake of clarity, we shall reserve the notation $\hat{P}, \hat{Q}$, etc. to refer to matrices in $\Delta_{1}$. 
Base: $p \in \Delta_{1}^{n}$. Trivial.

Step: $p \in \Delta_{j+1}^{n}$. Assuming that the lemma holds for all atomic matrices in $\Delta_{j}^{n}$ we show that it holds also for all atomic matrices in $\Delta_{j+1}^{n}$.

Suppose $p$ is an atomic matrix which belongs to $\Delta_{j+1}^{n}$ but does not belong to $\Delta_{j}^{n}$. Then, $p$ is non-null and, by Axiom 3, all atomic matrices in $\Delta_{j}^{n}$ are strictly less than $p$. Let now $q$ be a non-null atomic matrix in $\Delta_{j}^{n}$. Then, $q \prec_{M} p$ and, by Axiom 4 , $q^{m}+R \sim_{M} p^{m}$ for some $m \geq n$ and some non-null $R \in \mathcal{P}_{m}$. Since, $q^{m}$ is itself non-null, this implies (again by Axiom 4) that $R \prec_{M} p^{m}$. Now, we argue that $R$ must be in $\Delta_{j}^{m}$. We reason by absurd. Suppose $R \notin \Delta_{j}^{m}$, then $R=r+S$ for some atomic $r \in \mathcal{P}_{m}$ not in $\Delta_{j}^{m}$, and some (possibly empty) matrix $S \in \mathcal{P}_{m}$. However (by Axiom 3) $r \succeq_{M} p^{m}$ and (by Lemma 2) $r+S \succeq_{M} p^{m}$. Hence, $R \succeq_{M} p^{m}$ against the conclusion, reached before, that $R \prec_{M} p^{m}$. Thus, $R$ must be in $\Delta_{j}^{m}$ and so also $q^{m}+R$ is in $\Delta_{j}^{m}$. By inductive hypothesis, there is an $m^{\prime} \geq m$ such that $q^{m^{\prime}}+R^{m^{\prime}} \sim_{m} \hat{P}$ for some $\hat{P} \in \Delta_{1}^{m^{\prime}}$. So, since $p^{m} \sim_{m} q^{m}+R$, by Lemma $1, p^{m^{\prime}} \sim_{M} \hat{P}$. This concludes the proof of the lemma.

\section{B.2. Proof of the main theorem.}

Proof. We leave it to the reader to prove that the ordering satisfies Axioms 1-4.

To show that any ordering $\preceq_{M}$ which satisfies the axioms must be of the required form, let $P, Q$ be two matrices in $\mathcal{P}_{n}$. First, recall that $P$ and $Q$ can be rewritten as sums of atomic matrices,

$$
P=\sum_{(i, j) \in S(P)} p_{(i, j)} \text { and } Q=\sum_{(i, j) \in S(Q)} p_{(i, j)}
$$

where $p_{(i, j)}$ is the atomic matrix in $\mathcal{P}_{n}$ such that $S\left(p_{(i, j)}\right)=\{(i, j)\}$.

By Lemma 6, for each atomic $p_{(i, j)} \in \mathcal{P}_{n}$, there is an $k \geq n$ such that $p_{(i, j)}^{k} \sim_{M} \hat{P}_{(i, j)}$ for some $\hat{P}_{(i, j)}$ in $\Delta_{1}^{k}$. Observe that, by Remark 1 , one can always find, for each $(i, j) \in S(P) \cup S(Q)$, a suitable matrix $\hat{P}_{(i, j)}^{\prime}$ in $\Delta_{1}^{K}$, for some sufficiently large $K$, such that (i) $\hat{P}_{(i, j)}^{\prime}$ is atomically equivalent to $\hat{P}_{(i, j)}^{K}$ (and therefore, by Lemma 1, also to $\left.p_{(i, j)}^{K}\right)$, (ii) all the $\hat{P}_{(i, j)}^{\prime}$ such that $(i, j) \in S(P)$ are mutually disjoint, and (iii) all the $\hat{P}_{(i, j)}^{\prime}$ such that $(i, j) \in S(Q)$ are mutually disjoint.

Thus, by Axiom 2,

$$
\begin{aligned}
P^{K} & =\sum_{(i, j) \in S(P)} p_{(i, j)}^{K} \sim_{M} \sum_{(i, j) \in S(P)} \hat{P}_{(i, j)}^{\prime} \\
Q^{K} & =\sum_{(i, j) \in S(Q)} p_{(i, j)}^{K} \sim_{M} \sum_{(i, j) \in S(Q)} \hat{P}_{(i, j)}^{\prime} .
\end{aligned}
$$


Hence, for all $P, Q \in \mathcal{P}_{n}$

$$
P \preceq_{M} Q \Longleftrightarrow P^{K} \preceq_{M} Q^{K} \Longleftrightarrow \sum_{(i, j) \in S(P)} \hat{P}_{(i, j)}^{\prime} \preceq_{M} \sum_{(i, j) \in S(Q)} \hat{P}_{(i, j)}^{\prime}
$$

Now, let

$$
\mathcal{S}_{(i, j)}^{k}=\left\{Q \in \Delta_{1}^{k} \mid Q \sim_{M} p_{(i, j)}^{k}\right\}
$$

and

$$
\mathcal{S}_{(i, j)}=\bigcup_{k \in \mathbb{N}} \mathcal{S}_{(i, j)}^{k}
$$

We show that any two matrices in $\mathcal{S}_{(i, j)}$ contain the same number of non-null atomic matrices, and therefore this number depends only on $i$ and $j$. Let $Q_{1}$ and $Q_{2}$ be any two matrices in $\mathcal{S}_{(i, j)}$. Then, for some $k, k^{\prime}, Q_{1} \in \Delta_{1}^{k}, Q_{2} \in \Delta_{1}^{k^{\prime}}, Q_{1} \sim_{M} p_{(i, j)}^{k}$ and $Q_{2} \sim_{M}$ $p_{(i, j)}^{k^{\prime}}$. We assume without loss of generality that $k^{\prime} \geq k$. By Lemma $1, p_{(i, j)}^{k^{\prime}} \sim Q_{1}^{k^{\prime}}$, and so $Q_{1}^{k^{\prime}} \sim_{M} Q_{2}$. Since these two matrices are both in $\Delta_{1}^{k^{\prime}}$, by Lemma 5 , they must contain the same number of non-null atomic matrices. Moreover, the number of nonnull atomic matrices contained in $Q_{1}^{k^{\prime}}$ is the same as the number of those contained in $Q_{1}$. Thus, all the matrices in the set $\mathcal{S}_{(i, j)}$ contain the same number of non-null atomic matrices which depends only on $i$ and $j$. Let us denote it by $n_{(i, j)}$ and let $f$ be the function $\mathbb{N} \mapsto \mathbb{N}$ such that, for every $i, j, f(|i-j|)=n_{(i, j)}$. So, since $P_{(i, j)}^{\prime}$ belongs to $\mathcal{S}_{(i, j)}$, the number of non-null atomic matrices contained in $P_{(i, j)}^{\prime}$ is equal to $f(|i-j|)$.

Now, the matrices $\sum_{(i, j) \in S(P)} \hat{P}_{(i, j)}^{\prime}$ and $\sum_{(i, j) \in S(Q)} \hat{P}_{(i, j)}^{\prime}$ in $(3)$ are in $\Delta_{1}^{K}$. So, by Lemma 5 , they can be compared by simply counting the number of non-null atomic matrices contained in them. This is equal to the sum of the numbers of non-null atomic matrices contained in each $P_{(i, j)}^{\prime}$ which is, in turn, equal to $f(|i-j|)$. Therefore:

$$
\sum_{(i, j) \in S(P)} \hat{P}_{(i, j)}^{\prime} \preceq_{M} \sum_{(i, j) \in S(Q)} \hat{P}_{(i, j)}^{\prime} \Longleftrightarrow \sum_{(i, j) \in S(P)} f(|i-j|) \leq \sum_{(i, j) \in S(Q)} f(|i-j|) .
$$

Finally, from (3) and (4) it follows that:

$$
P \preceq_{M} Q \Longleftrightarrow \sum_{(i, j) \in S(P)} f(|i-j|) \leq \sum_{(i, j) \in S(Q)} f(|i-j|) .
$$

It is obvious, by Axiom 3, that $f$ must be strictly increasing. To show that $f$ must be strictly convex, consider, for any $k \geq 0$, the matrices $P$ and $Q$ such that:

$$
\begin{aligned}
& S(P)=\{(1, k+1),(2, k+2),(k+3, k+3),(k+4, k+4), \cdots\} \\
& S(Q)=\{(1, k+2),(2, k+1),(k+3, k+3),(k+4, k+4), \cdots\} .
\end{aligned}
$$

Hence, by Axiom 1, we must have that, for all $k, 2 f(k)<f(k+1)+f(k-1)$. 


\section{Appendix C. Proof of Theorem 3}

Proof. Given Theorem 2, we can concentrate only on Axiom 5. The reader can easily check that if $f(k)=k^{2}$ the ordering satisfies Axiom 5 .

To show that, in order to satisfy Axiom $5, f$ must be quadratic, suppose $P, Q, R$ are matrices in $\mathcal{P}_{n}$ such that:

$$
\begin{aligned}
& S(P)=\{(0,0),(1,1),(0, k),(1, k+1)\} \\
& S(Q)=\{(0,1),(1,0),(0, k),(1, k+1)\} \\
& S(R)=\{(0,0),(1,1),(0, k+1),(1, k)\} .
\end{aligned}
$$

Thus, $P \triangleleft Q$ and $P \triangleleft R$, since the inversions that lead from $P$ to $Q$ and from $P$ to $R$ are both minimal. Then, by Axiom 5, $Q \sim_{M} R$ and therefore:

$\sum_{(i, j) \in S(Q)} f(|i-j|)=2 f(1)+2 f(k)=\sum_{(i, j) \in S(R)} f(|i-j|)=2 f(0)+f(k+1)+f(k-1)$.

Observe that, by definition of $f, f(0)=0$, and $f(1)=1$ (see above, Appendix B.2). Therefore, to satisfy Axiom 5 , since $k$ is arbitrary and $f$ is fixed for all $n$, we must have that, for all $k$,

$$
f(k+1)-f(k)=f(k)-f(k-1)+2 .
$$

This difference equation has a unique solution, i.e. $f(k)=k^{2}$.

\section{REFERENCES}

[Atk83] A.B. Atkinson. The measurement of economic mobility. In A.B. Atkinson, editor, Social Justice and Public Policy. Wheatsheaf Books Ltd., London, 1983.

[BCFS90] H.W. Block, D. Chhetry, Z. Fang, and A.R. Sampson. Partial orders on permutations and dependence orderings on bivariate empirical distributions. The Annals of Statistics, 18:1840-50, 1990.

[BFFK05] M. Buchinsky, G. Fields, D. Fougre, and F. Kramarz. Francs and ranks: Earnings mobility in france, 1967-1999. CEPR Discussion Paper, 9, 2005.

[CBW85] S.R. Chakravarty, Dutta B., and J. A. Weymark. Ethical indices of income mobility. Social Choice and Welfare, 2:1-21, 1985.

[Cow85] F. Cowell. Measures of distributional change: An axiomatic approach. Review of Economic Studies, 52:35-51, 1985.

[Dar93] V. Dardanoni. Measuring social mobility. Journal of Economic Theory, 61:372-94, 1993.

[ET80] L.G. Epstein and S.M. Tanny. Increasing generalized correlation: A definition and some economic consequences. Canadian Journal of Economics, 13:16-34, 1980.

[Fie02] G. S. Fields. Distribution and Development: A New Look at the Developing World. MIT Press, Boston, 2002.

[FO96] G.S. Fields and E. Ok. The meaning and measurement of income mobility. Journal of Economic Theory, 71:349-77, 1996.

[FO99a] G.S. Fields and E. Ok. The measurement of income mobility. In J. Silber, editor, Handbook of Income Inequality Measurement. Kluwer Academic Publishers, Dordrecht, 1999. 
[FO99b] G.S. Fields and E. Ok. Measuring movements of incomes. Economica, 66:455-71, 1999.

[FS91] J.E. Foster and A.F. Shorrocks. Subgroup consistent poverty indices. Econometrica, 59:687-709, 1991.

[FS97] J.E. Foster and A. Sen. On economic inequality after a quarter century. In A. Sen, editor, On Economic Inequality. Clarendon Press, Oxford, 1997.

[GS02] P Gottschalk and E. Spolaore. On the evaluation of economic mobility. Review of Economic Studies, 69:191-208, 2002.

[HJ91] R. A. Horn and C. R. Johnson. Topics in Matrix Analysis. CUP, 1991.

[KG90] M. Kendall and J.D. Gibbons. Rank Correlation Methods. Edward Arnold, 1990.

[Kin83] M.A. King. An index of ineqaulity: with applications to horizontal equity and social mobility. Econometrica, 51:99-116, 1983.

[KS87] G. Kimeldorf and A.R. Sampson. Positive dependence orderings. Annals of the Institute of Statistical Mathematics, 39:113-128, 1987.

[Maa98] E. Maasoumi. On mobility. In D. Giles and A. Ullah, editors, The Handbook of Economic Statistics. Marcel Dekker, New York, 1998.

[MO98] T. Mitra and E. Ok. The measurement of income mobility: A partial ordering approach. Economic Theory, 12:77-102, 1998.

[MZ86] E. Maasoumi and S. Zandvakili. A class of generalized measures of mobility with applications. Economics Letters, 22:97-102, 1986.

[Nel99] R. B. Nelsen. An Introduction to Copulas. New York: Springer, 1999.

[RC04] J. Ruiz-Castillo. The measurement of structural and exchange mobility. Journal of Economic Inequality, 2:219-228, 2004.

[Sho88] A.F. Shorrocks. Aggregation issues in inequality measurement. In W. Eichorn, editor, Measurement in Economics. Springer Verlag, New York, 1988.

[SW81] B. Schweizer and E.F. Wolff. On non-parametric measures of dependence for random variables. Annals of Statistics, 9:879-885, 1981.

[Tch80] A.H.T. Tchen. Inequalities for distributions with given marginals. Annals of Probability, 8:814-27, 1980. 